UCRL-JC-121540

PREPRINT

$$
\text { CONF-951155- - 77 }
$$

\title{
A Phenomenological Approach to Simulating the Evolution of Radioactive-Waste Container Damage Due to Pitting Corrosion
}

\author{
Gregory A. Henshall
}
RECFIVED
MAR 011996
OSTI

This paper was prepared for submittal to the

Materials Research Society's Fall Meeting

Boston, MA

November 27 - December 1, 1995

October 25, 1995

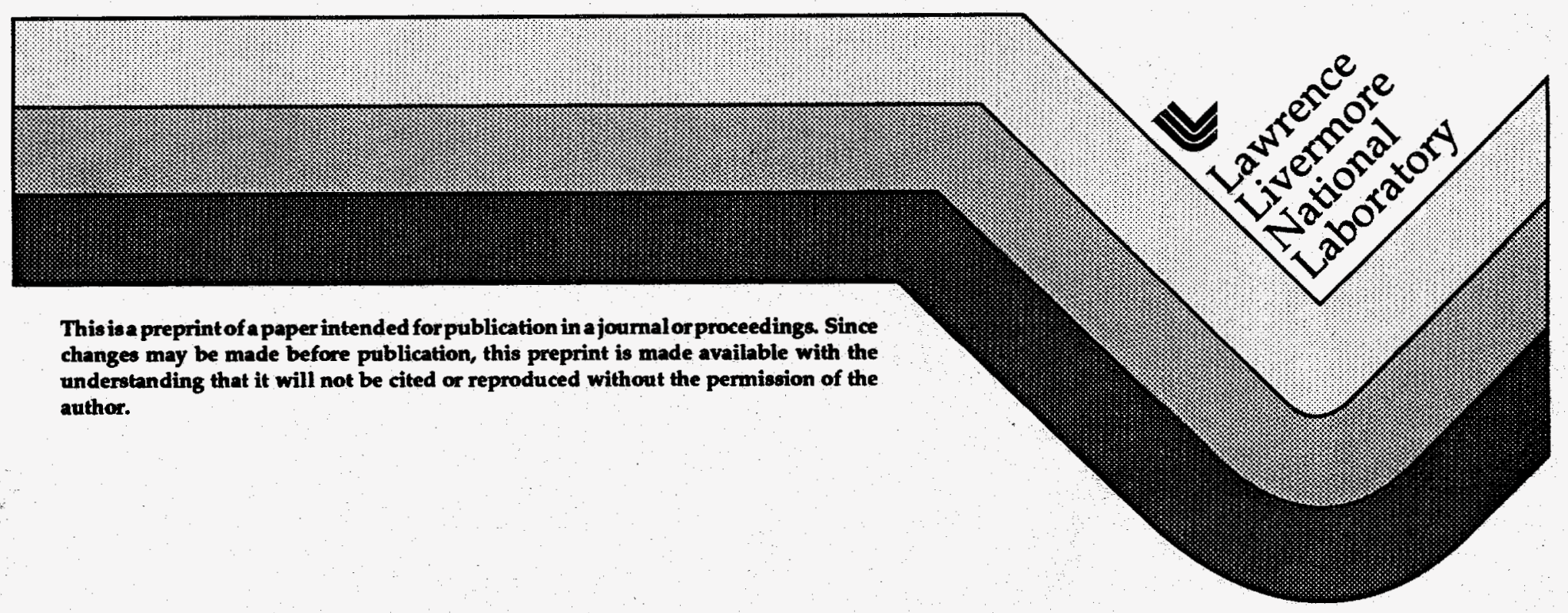




\section{DISCLAIMER}

This decument was prepared as an account of work sponsored by an agency of the United States Government. Neither the United States Government nor the University of Cdifornia nor any of their employees, makes any warranty, express or implied, or assumes any legal liability or responsibility for the nocuracy, completeness, or usefulness any information, apparatus, product, or process disclosed, or represents that its use would not infringe privalely owned rights. Reference herein to any specific commercial products, process, or service by trade name, trademark, manufacturer, or otherwise, does not necessarily constitute or imply its endorsement, recommendation, or favoring by the United States Government or the University of Califomia. The views and epinions of authors expressed herein do not necessarily state or reflect those of the United States Government or the University of Califomia, and shall not be used for advertising or produet endorsement purposes. 


\section{DISCLAIMIER}

Portions of this document may be illegible in electronic image products. Images are produced from the best available original document. 


\title{
A PHENOMENOLOGICAL APPROACH TO SIMULATING THE EVOLUTION OF RADIOACTIVE-WASTE CONTAINER DAMAGE DUE TO PITTING CORROSION
}

Gregory A. Henshall, Lawrence Livermore National Laboratory, Livermore, CA.

\begin{abstract}
The damage to high-level radioactive-waste containers by pitting corrosion is an important design and performance assessment consideration. It is desirable to calculate the evolution of the pit depth distribution, not just the time required for initial penetration of the containers, so that the area available for advective or diffusive release of radionuclides through the container can be estimated. A phenomenological approach for computing the time evolution of these distributions is presented which combines elements of the deterministic and stochastic aspects of pit growth. The consistency of this approach with the mechanisms believed to control the evolution of the pit depth distribution is discussed. Qualitative comparisons of preliminary model predictions with a variety of experimental data from the literature are shown to be generally favorable. The sensitivity of the simulated distributions to changes in the input parameters is discussed. Finally, the results of the current model are compared to those of existing approaches based on extreme-value statistics, particularly regarding the extrapolation of laboratory data to large exposed surface areas.
\end{abstract}

\section{INTRODUCTION}

The damage to high-level radioactive-waste containers by pitting corrosion is an important engineered barrier system (EBS) design and performance assessment (PA) consideration. It has been suggested [1] that a statistical approach to characterizing and modeling localized corrosion, in contrast to uniform corrosion, is necessary even though the data requirements are large. One advantage of a statistical, or stochastic, method is that the evolution of the pit depth distribution, not just the time required for initial penetration of the containers, can be computed so that the area available for advective or diffusive release of radionuclides through the containers can be estimated [2]. This paper describes a phenomenological approach to the stochastic modeling of pitting corrosion damage and presents some predictions of pit depth distribution evolution from the preliminary model.

\section{MODELING APPROACH}

Following earlier work [3], Monte Carlo computer codes have been used to simulate the apparently random initiation of permanent pits on a unit surface area of metal in contact with an aggressive environment. In previous models, these permanent pits were assumed to grow an increment in depth during each simulated time step only if a random number, $0 \leq R<1$, is less than the growth probability $\gamma$, where $0 \leq \gamma \leq 1$. This approach led to the time evolution of a distribution of pit depths [3]. However, these distributions did not exhibit the positive skew frequently observed experimentally [4-7], in which the majority of pits have small depths and a long "tail" to the distribution exists at large depths. Motivated by the need to more accurately 
model this aspect of the pit depth distribution evolution, efforts to improve the pit growth model were made.

Revision of the pit growth model follows from a variety of observations reported in the literature. In a study of the pit depth distribution evolution in mild steel, Marsh et al. [4] identified four factors having the potential to produce the wide distribution in pit depths observed on any given sample:

1) The pits will have different initiation times.

2) Some pits may cease to propagate before the termination of the experiment.

3) The morphology of the pits will vary, with some favoring more rapid mass and charge transfer, and hence faster propagation rates.

4) Some pits will initiate at metallurgical features which may favor more rapid propagation, e.g. MnS inclusions.

Items (1) and (4) already are included in the phenomenological model through the stochastic treatment of permanent pit initiation [3]. Items (3) and (4) suggest that individual pits grow at a range of rates, which is simulated in the stochastic model through the growth probability per time step, $\gamma$ [3]. To accommodate observation (2), a new stochastic variable was introduced: the probability that during any given time step a pit will permanently cease to grow, $\eta$. Again, this probability is compared against a random number, $0 \leq R<1$, for each growing pit at each time step, and the pit is permanently halted if $R \leq \eta$.

Further support for the use of a stochastic growth probability, $\gamma$, and a permanent cessation probability, $\eta$, is given by the data and analysis of Aziz [7] for the pitting corrosion of aluminum in tap water. Figure 1 shows the pit depth distribution data of Aziz. When first exposed, a large number of pits initiate and start propagating. After a short time, many pits progressively stifle while only a portion of the population continues to grow, resulting in a backwards " $\mathrm{J}$ " shape to the low depth portion of the distribution (supports the concept of permanent pit growth cessation, $\eta$ ). For those pits still growing, the random influence of the environment on propagation rate results in a bell-shaped distribution, which moves as a body toward larger depths (supports the concept of stochastic pit growth, $\gamma$ ). After much longer exposures, the mode, i.e. peak, of the distribution becomes stationary (supports $\eta$ ) and only the deeper pits continue growing. These pits grow at a steadily decreasing rate (which supports the use of a nonlinear increase in pit depth with time introduced below), and the majority of the pits eventually stifle (again, supports $\eta$ ). The last two findings may result from the build-up of corrosion products within and over pits.

Despite the fact that pits grow to have a wide distribution of depths, indicating a probabilistic aspect to pit growth, many theories treat pit growth deterministically, typically as a problem in diffusion [8]. Further, diffusion theories and a variety of experimental observations, e.g. $[7,8]$, suggest that the pit growth rate decreases as the exposure time and pit depth increase. Equation (1) is often used to phenomenologically describe these deterministic aspects of pit growth (for constant environmental conditions): 


$$
d=A t^{p} ; p<1
$$

where $d$ is pit depth, $t$ is exposure time, and $A$ and $p$ are constants. Typically, $p<1$ with values near 0.5 being most common. Equation (1) has been incorporated into the revised stochastic model with one slight modification. Instead of representing the exposure time, $t$ is the pit "age", which is less than the actual exposure time for two reasons. First, it takes some "induction" time, $\tau$, to initiate a pit following exposure [3]. Second, pits are assumed to grow, or "age," stochastically with some probability $\gamma$, and may permanently cease to "age" with some probability $\eta$; i.e. pits do not age during all time steps. Thus, at the end of a simulation the pits have a distribution of "ages," from which the corresponding depths are computed using Eq. (1). Of course, Eq. (1) is not ideal for modeling purposes because it precludes easy treatment of changing environmental conditions. However, it is worthwhile to use this equation in combination with the concepts of stochastic pit growth and permanent cessation as a means to begin exploring models incorporating both the deterministic and probabilistic aspects of pit growth.

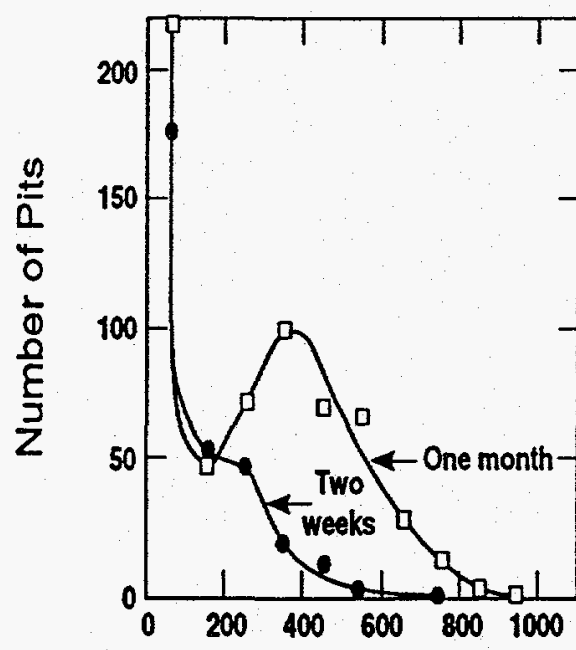

Pit Depth (um)

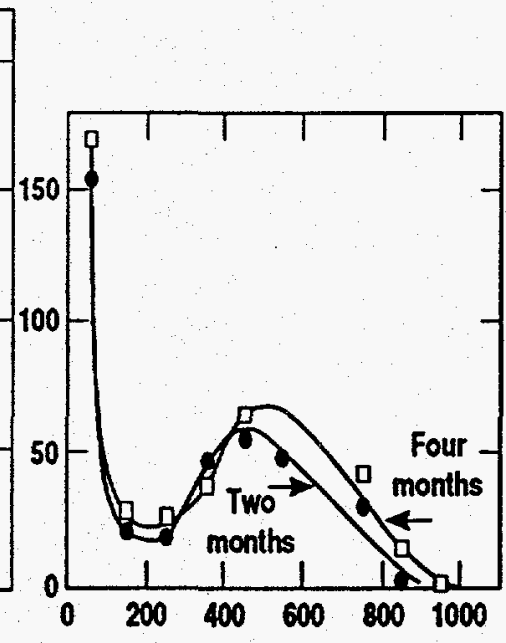

Pit Depth ( $\mu \mathrm{m})$

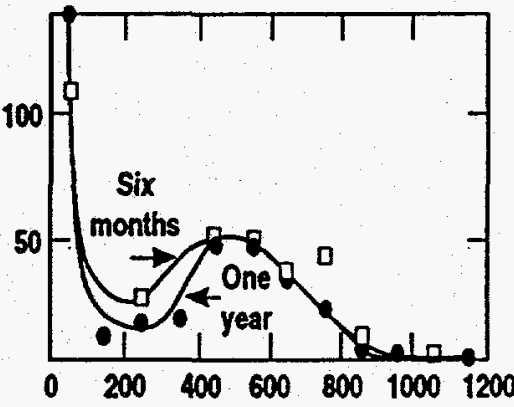

Pit Depth (um)

Figure 1. The distribution of pit depths as a function of exposure time for Alcan 2S-O aluminum immersed in tap water. Data of Aziz [7].

\section{MODEL PREDICTIONS}

Figure 2 illustrates the capability of the new model to simulate the complex time evolution of the pit depth distribution. For this simulation, the probability of initiating a new pit embryo was decreased exponentially with exposure time [3] such that no new pits were initiated for exposures greater than approximately 50 time steps. The parameters affecting the growth of these pits, $\gamma, \eta, A$ and $p$, were chosen arbitrarily and are given in the figure. At very short exposure times (less than 50 steps), the distribution in pit depths monotonically decays with increasing depth. As shown in Fig. 2, for longer exposures the distribution takes on shapes qualitatively similar to those observed by Aziz [7], Fig. 1. Figure 2(a) exhibits a peak 
in the distribution at the lowest depths, followed by a decrease in the number of pits with increasing depth, and then a second local maximum. This gives the backwards " $J$ " shape to the initial part of the distribution noted by Aziz. Experimentally, this portion of the distribution was attributed to the stifling of shallow pits. In the model, this same feature is caused by the permanent cessation of growth for many small pits through the parameter $\eta$. As the exposure time increases, the heights of the two peaks decrease somewhat as the distribution broadens, Fig. 2(b). The broad distribution of pit depths, particularly beyond the backwards " $\mathrm{J}$ " feature, was attributed by Aziz [7] largely to the randomly varying propagation rates for individual pits. This same characteristic is predicted by the model largely through the stochastic growth probability, $\gamma$. With further increases in exposure time, the two peaks remain stationary and only the deepest pits continue to grow, creating a long "tail" to the distribution, Fig. 2(c). This same feature in the data of Aziz, Fig. 1, is caused by the eventual stifling of most pits, so that only a few grow to large depths. Consistent with this finding, the growth cessation probability, $\eta$, causes most pits to stop growing after 500 time steps. Finally, note in Fig. 1 that the maximum pit depth, $d_{\max }$, increases ever more slowly as the exposure time increases. A similar phenomenon is evident in Fig. 2. Following 100 time steps $d_{\max }=3.5$, while after a five-fold increase in exposure time $d_{\text {max }}$ has less than doubled to 5.5, Fig. $2(\mathrm{c})$. This prediction results partly from the use of Eq. (1) with $p=0.5$. In addition, the rate of increase for $d_{\max }$ slows due to the use of $\eta=0.01$. Consider that for an exposure of 500 steps only four of the initial 590 pits are still growing. This implies that the pits which early in the exposure were of maximum depth probably have permanently halted, requiring shallower pits to grow and become the deepest active pits at longer exposures. In summary, the complex pit depth distribution evolution exhibited in Fig. 1 is qualitatively predicted by the model through the consideration of both the stochastic and deterministic aspects of pit growth.

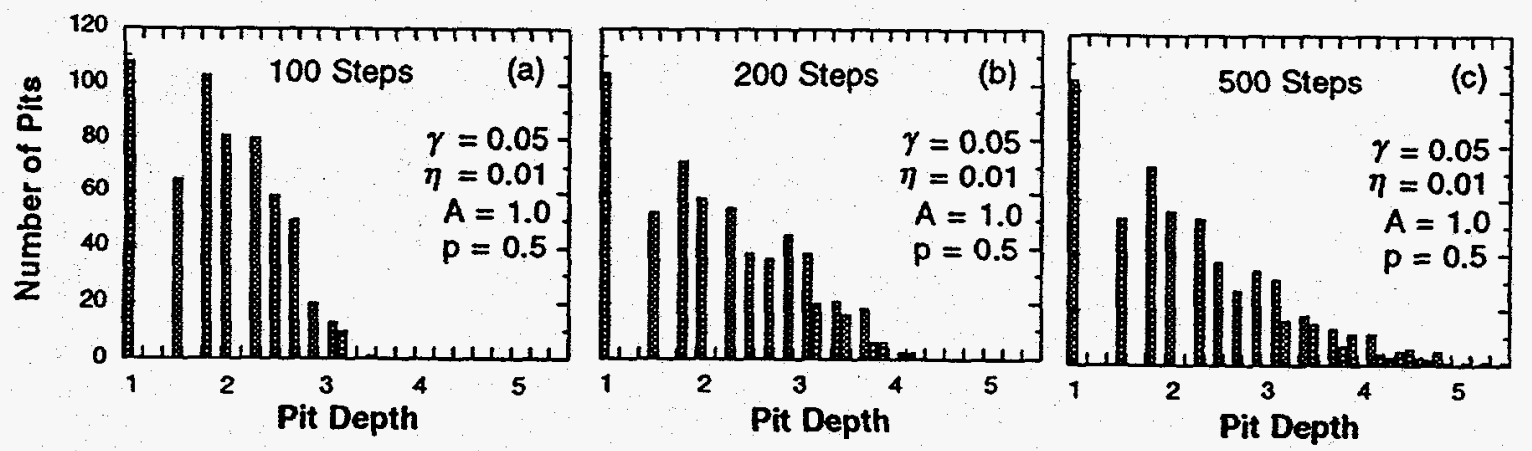

Figure 2. Computed distribution in pit depths for the growth parameters given in the figure and exposures of: (a) 100 time steps, (b) 200 time steps, and (c) 500 time steps. Pit depths are in arbitrary units.

Of course, the evolution of the pit depth distribution is sensitive to the input parameters affecting pit growth. Calculations have shown that the shapes of the distributions during various stages of evolution are most sensitive to the relative values of the pit growth and permanent pit cessation probabilities, $\gamma$ and $\eta$. For example, decreasing the value of $\eta$ by a 
factor of 10 below that used in the calculations presented in Fig. 2 (while maintaining $\gamma$ constant) leads to a dramatic change in the distribution evolution. Similar to Fig. 2, Fig. 3 shows the results of these calculations following relatively short, intermediate, and long exposures. (For $\eta=0.01$, Fig. 2, nearly all of the pits had ceased to grow after 500 time steps. For $\eta=0.001$, Fig. 3, a similar level of halted pits is not reached until exposures of approximately 7500 time steps.) For short exposures, Fig. 3(a), decreasing $\eta$ to 0.001 results in a loss of the backwards " $\mathrm{J}$ " shape at small pit depths that was present in the corresponding distribution for $\eta=0.01$. Following intermediate exposures, decreasing $\eta$ to 0.001 produces a long "plateau" in the number of pits at small depths, which did not result from the calculations with $\eta=0.01$. Such a plateau, Fig. 3(b), has not been observed experimentally but, as pointed out by Marsh et al. [4], the smallest pits often cannot be accurately measured. Therefore, it is not clear whether or not the distribution in Fig. 3(b) is unrealistic. Following long exposures, Fig. 3(c), decreasing $\eta$ to 0.001 results in a distribution similar to that shown in Fig. 2(c), except that the peak at very small pit depths in Fig. $2(\eta=0.01)$ is not predicted.
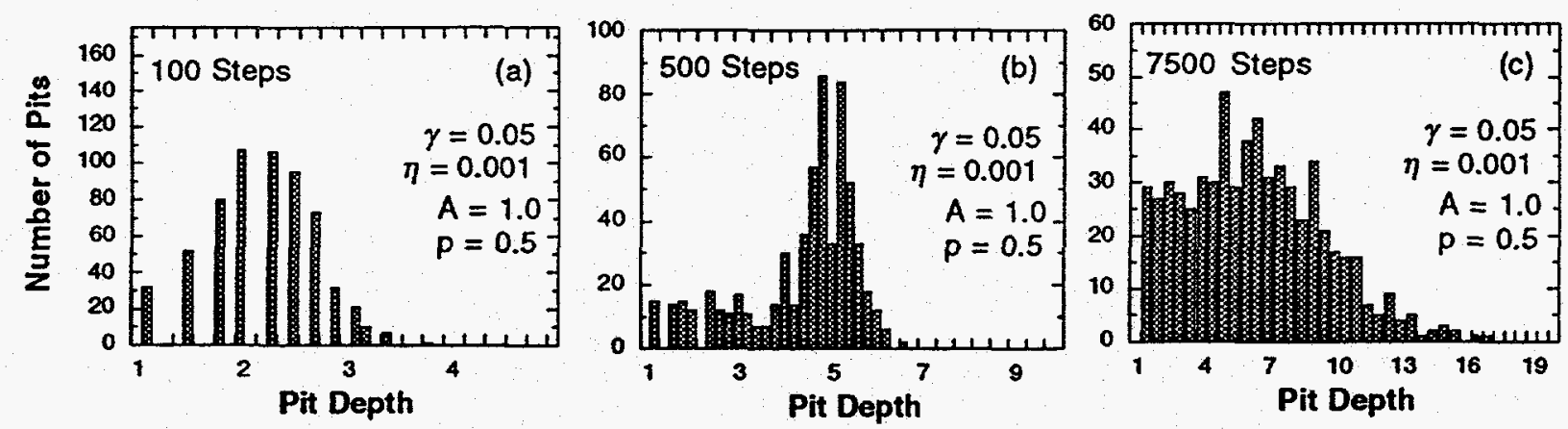

Figure 3. Computed distribution in pit depths for the growth parameters given in the figure and exposures of: (a) 100 time steps, (b) 500 time steps, and (c) 7500 time steps. Pit depths are in arbitrary units.

\section{COMPARISON WITH EXTREME-VALUE STATISTICAL METHODS}

The method of "extreme-value statistics" (EVS) is commonly employed in the analysis of experimental pit depth data, e.g. [7,9-11]. It has been shown that this method is particularly valuable for predicting the logarithmic increase in maximum pit depth with increasing specimen or service component surface area which has been observed experimentally $[4,6,7,11]$. In the context of EBS design and PA, EVS analysis of laboratory data collected on a limited number of small specimens might provide the means to predict the probability for the existence of a pit of any given depth (e.g. one equal to the container thickness) over the surface of an entire container, or many containers. Such a prediction should be accurate so long as the extrapolated pit depth exceeds the deepest measured pit by no more than about a factor of 3 [7]. Fortunately, this provides an extrapolation in exposed surface area of up to three orders of magnitude.

Given the utility and wide acceptance of EVS to analyze pitting damage, it useful to determine if the stochastic pitting model is consistent with this method. Therefore, 
modifications to the Monte Carlo code were made following the actual experimental procedure described by Aziz [7]. First, an individual test coupon is simulated by a single model "run" using a unique "seed" value to initiate the random number generator [3]. Using a single set of input parameters, an $N$-run simulation is performed in which each run begins with a different random number seed. This procedure corresponds to the exposure of multiple, identical test coupons to the same corrosive environment, as described by Aziz [7]. Analogous to the experiments, the surface area simulated is proportional to the number of runs in the simulation, since one run considers a unit surface area. Following each run within a particular $N$-run simulation, the maximum computed pit depth is stored. Once all $N$ runs have been completed, these values are sorted in ascending order, providing "data" analogous to that collected experimentally [7]:

$$
d_{1}^{\max } \leq d_{2}^{\max } \leq \ldots \leq d_{m}^{\max } \leq \ldots \leq d_{N}^{\max }
$$

The cumulative probability that the deepest pit is less than or equal to $d_{m}^{\text {max }}$ is then computed from these data as:

$$
\Phi_{m}=m /(N+1)
$$

These probabilities from the Monte Carlo simulation are then analyzed using the most common expression for the expected extreme-value distribution [7]:

$$
\Phi_{m}=\exp \left[-\exp \left(-y_{m}\right)\right]
$$

where $y_{m}$ is the "reduced variate." The reduced variate is defined as:

$$
y_{m}=\alpha\left(d_{m}^{\max }-u\right)
$$

where $u$ is the "mode" (highest point of the extreme-value distribution) and $\alpha$ is the "scale" parameter measuring the width of this distribution. The values of $u$ and $\alpha$ are fit to the Monte Carlo "data" [Eq. (2)] as follows. First, Eq. (4) is solved for $y_{m}$, giving:

$$
y_{m}=-\ln \left\{-\ln \left(\Phi_{m}\right)\right\}
$$

where $\Phi_{m}$ is computed from the Monte Carlo results using Eq. (3). For each value of $\Phi_{m}$, a corresponding $y_{m}$ is computed from Eq. (6). Then, the data pairs $\left(d_{m}{ }^{\max }, y_{m}\right)$ are plotted on linear axes and fit to Eq. (5) using linear least squares analysis to determine $\alpha$ and $u$. Extrapolation of this fitted line provides predictions of the probability and exposure time required for the occurrence of a pit of any given depth [7]. 
In general, the stochastic model is fairly consistent with the EVS theory. For example, Fig. 4 shows the results of a 50-run simulation in which each run consisted of 500 time steps of exposure. The consistency between the simulation (solid circles) and the linear least squares fit (solid line) is typical of that observed in actual experiments by Aziz for $\mathrm{Al}$ in tap water [7]. The reasonably good linear fit to the Monte Carlo results indicates that the extreme pit depth values predicted by the stochastic model behave in a manner similar to those measured experimentally in several material/environment systems [7,9-11]. Further, consistent with previous EVS analyses and data $[4,6,7,11]$, the stochastic model predicts that the maximum pit depth increases logarithmically with the exposed surface area, Fig. 5. Thus, by performing simulations with identical input parameters and varying the numbers of runs, the effect of exposed container surface area on the maximum pit depth can be predicted by the stochastic model.

4)

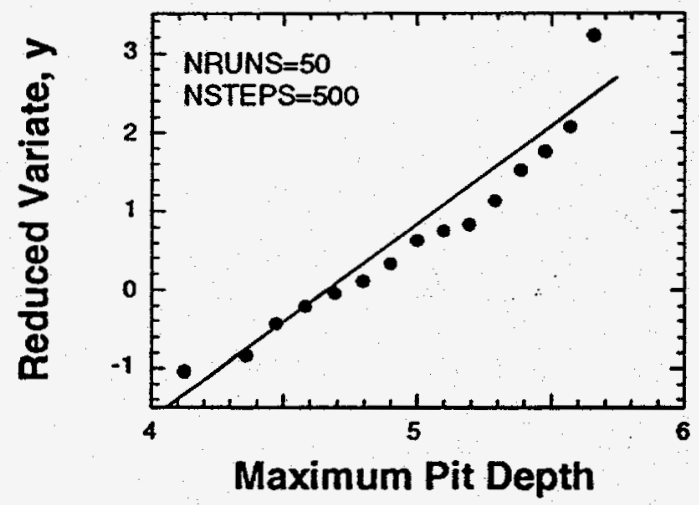

5)

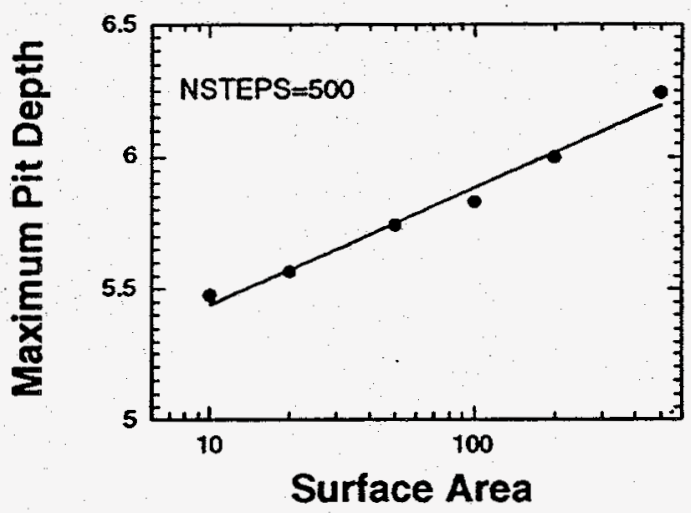

Fig. 4. The reduced variate as a function of maximum pit depth (arbitrary units) computed by the stochastic model (solid circles) is compared with the linear least squares fit (solid line) for a 50-run simulation with 500 time steps per run.

Fig. 5. The maximum pit depth as a function of the exposed surface area (equal to the number of runs per simulation) predicted by the stochastic model. Both maximum pit depth and surface area are in arbitrary units.

\section{CONCLUSIONS}

A phenomenological stochastic model of pit initiation and growth has been developed. This model can simulate the time evolution of the distribution in pit depths on a metal surface exposed to an aggressive environment. Pit growth is simulated as a stochastic process in which the growth of individual pits during a unit time is controlled by a pit growth probability, $\gamma$, and a permanent pit cessation probability, $\eta$. The use of these two parameters is motivated by experimental investigations demonstrating that pits grow at a distribution of rates and that many pits permanently stop growing during exposure. Finally, a deterministic aspect of pit growth is included in the model by assuming that the pit depth increases more slowly with 
increasing pit "age" according to a simple power law. Including these three aspects of pit growth, together with stochastic pit initiation, provides for the capability to qualitatively simulate many aspects of the time evolution of the pit depth distribution. An initial parameter sensitivity study shows that the evolution of these distributions is most sensitive to the relative values of $\gamma$ and $\eta$, and less sensitive to other input parameters. Further, this model has been shown to be consistent with extreme-value statistical methods for predicting the effect of surface area on the expected maximum pit depth. Experimental data are required to quantitatively assess and further develop the model for nuclear-waste container candidate materials exposed to relevant environments.

\section{ACKNOWLEDGMENTS}

This work was performed under the auspices of the U. S. Department of Energy under contract No. W-7405-ENG-48 at the Lawrence Livermore National Laboratory. This work was supported by the Yucca Mountain Site Characterization Project.

\section{REFERENCES}

1. D. Shoesmith, "Modelling to Predict the Corrosion Behavior of Nuclear Waste Containers," presented at the Forum for the Use of Nickel Alloys for Radwaste Containers, Tucson, AZ, Feb. 22-23 (1995).

2. R. W. Andrews, "TSPA-95 Objectives and Approach: Focus on Waste Packages/EBS Conceptual Models," presented at the NWTRB Full Board Meeting, Las Vegas, NV, Apr. 1920, (1995).

3. G. A. Henshall, "Modeling Pitting Corrosion Damage of High-Level Nuclear-Waste Containers using a Stochastic Approach," J. Nucl. Mater. 195, 109 (1992).

4. G. P. Marsh, I. D. Bland, and K. J. Taylor, "Statistical Study of Pit Propagation in Carbon Steel Under Nuclear Waste Disposal Conditions," Br. Corros. J. 23, 157-164 (1988).

5. V. Y. Flaks, "Statistical Model of Size Distribution of Pittings During Atmospheric Corrosion of Aluminum Alloys," Protection of Metals 9, 407-409 (1973).

6. J. W. Provan and E. S. Rodriguez, "Part I: Development of a Markov Description of Pitting Corrosion," Corros. 45, 178-192 (1989).

7. P. M. Aziz, "Application of the Statistical Theory of Extreme Values to the Analysis of Maximum Pit Depth Data for Aluminum," Corros. 12, 495-506 (1956).

8. A. Hoch, et al., "A Modelling and Experimental Study for Long-Term Prediction of Localised Corrosion in Carbon Steel Overpacks for High-Level Radioactive Waste," in MRS Symposium on the Scientific Basis for Nuclear Waste Management XVIII, pp. 703-710 (1995).

9. J. E. Strutt, J. R. Nicholls, and B. Barbier, "The Prediction of Corrosion by Statistical Analysis of Corrosion Profiles," Corros. Sci. 25, 305-315 (1985).

10. H. F. Finley, "An Extreme-Value Statistical Analysis of Maximum Pit Depths and Time to First Perforation," Corros. 23, 83-87 (1967).

11. G. G. Eldredge, "Analysis of Corrosion Pitting by Extreme-Value Statistics and its Application to Oil Well Tubing Caliper Surveys," Corros. 13, 51-60 (1957). 